Aus dem Institut für Humangenetik der Universität Münster

Direktor: Prof. Dr. Frhr. v. Verschuer

\title{
MIGRÄNE BEI ZWILLINGEN, VORLÄUFIGE MITTEILUNG ${ }^{1-2}$ \\ von \\ H. C. Ebbing
}

Die Migräne ist eine psychophysische Reaktionsform auf zahlreiche Einzelreize. Nosologisch ist sie daher immer Symptom einer pathologischen Konstellation, die als "klassisches Beispiel einer Organneurose" ( $H$. Curschmann) eine Antwort darstellt auf sehr unterschiedliche Einwirkungen. Für die Manifestation ist somit eine Addition verschiedener pathogener Situationen oder "Konstellationen" (F. Mauz) erforderlich. Nach P. Matzdorff sind mindestens zwei Teilursachen erforderlich: eine organische oder funktionelle Störung im Hirn und eine humorale Dysregulation, i. e. " eine individuelle Ansprechbarkeit der endokrin-vegetativ gesteuerten Person » (H. Pette). «Die Migränekranken stellen also gewissermassen den Schnittpunkt mehrerer Teilbereitschaften dar» (F. Curtius).

Das «klassische" Bild der Hemikranie (Aretaeus von Cappadocia, Galen) trägt Anfallcharakter und wird oft durch eine vegetative Aura eingeleitet. Der halbseitige Kopfschmerz ist das nicht immer obligate Hauptsymptom. Nach H. G. Wolff ist der adaequate Reiz für eine Schmerzempfindung die Gewebsschädigung. Reaktionen auf diese Einwirkungen sind abhängig von dem modifizierenden Einfluss der höheren Zentren (Cortex). Der Kopschmerz darf als Symptom einer Reizung der vegetativen Rezeptoren in den Hirngefässen angesehen werden.

Neben der beim individuellen Krankheitserleben im Vordergrund stehenden Cephalgie oder ihrer Aequivalente stehen nosologisch gleichwertig andere Störungen des Vegetativums: Dysegulationen des Schlaf-Wachrhythmus, Uebelsein bis Erbrechen, paroxysmale Harnflut, Tachy- oder Bradykardie, Tachypnoe bis zur Hyperventilationstetanie u. a. Es wird über Störungen in der Blutzuckerregulation berichtet (J. Girard et al.). M. Kibler beobachtete Schwankungen der Körpertemperatur bei einem migränekranken Patienten, dessen Sohn gleichartig reagierte. Kibler weist darauf hin, dass diese Symptome dem Ergebnis der Katzenversuche von R. W. Hess entsprechen, bei denen einzelne Punkte des Diencephalon gereizt worden sind.

1 Die Untersuchung der Zwillinge wurde durch die Akademie der Wissenschaften und der Literatur (Mainz) ermöglicht.

2 Herrn Prof. Dr. med. O. Frhr. v. Verschuer zum 60. Geburtstag gewidmet. 
Die Analogie von Klinik und Experiment zeigt hin auf das Zwischenhirn, dem Zentrum vegetativer Regulationen. Weil sich im vegetativen System Peripherie und zentrale Schaltstelle wechselseitig beeinflussen, entsteht ein circulus vitiosus, der auch den Paroxysmus des Migräneanfalls erklärt. So ist es verständlich, dass es neben " genuinen » Formen anfallartige Reizzustände des Vegetativums als "symptomatische" Krankheitsbilder bei funktionellen oder organischen Laesionen der Peripherie geben kann.

Nach R. Jansen wird die Diagnose der genuinen Migräne per exclusionem gestellt. Die Differentialdiagnose erfolge nicht aus dem Syndrom, sondern wie bei der essentiellen Epilepsie durch die Analyse der Begleitumstände. Voraussetzung für das Wirksamwerden des in beiden Fällen notwendigen auslösenden Reizes ist die endogene Krampfbereitschaft. Wie Gerhard Koch in seiner Monographie für die Epilepsie dargelegt hat, handelt es sich dabei offenbar um vorgebildete Mechanismen, die durch genuine oder symptomatische, zentrale oder periphere Vorgänge ausgelöst werden können. In diesem Zusammenhang sei darauf hingewiesen, dass sowohl der erst einmal ausgelöste epileptische Anfall als auch der paroxysmale Kopfschmerz zu einem "Engramm" (J. Wagner von Jauregg) oder einem "gebahnten Reflex» (H. Pette) werden kann, der bei geringfügigen Anlässen ausgelöst wird. Das Beispiel des Allergiekranken von Zbinden, der auf den Anblick eines Phantoms mit einem schweren Anfall reagierte, macht die Bedeutung der gebahnten Reflexe deutlich.

Nach den bisherigen Familienuntersuchungen ist die Migräne eine erbbedingte Krankheit. Von 56 Elternpaaren, bei welchen beide Partner an Hemikranie litten, hatten nach W. Allan unter den 318 Kindern $83 \%$ ebenfalls anfallartige Kopfschmerzen. 750 Kinder mit einseitiger erblicher Belastung aus 141 Elternpaaren zeigten die Manifestation zu 61\%, während von 485 Nachkommen aus 98 Ehen gesunder Eltern nur 3,7\% erkrankt waren. Nach einer Zusammenstellung von Liselotte Eichler-Oheim fanden Symmonds et al. in 50\%, Bassoe in 56\%, Moebius in 90\%, Heyerdahl in $85 \%$, E. Mendel in $80 \%$ und Möhlig in 39\% ihrer Probanden eine gleichartige Erkrankung in der Sippe. Von den Kranken K. Grunnerts und P. Matzdorffs dagegen waren nur 20\% durch Migräne in der Blutsverwandtschaft belastet. Grunnert selbst hält jedoch diese Werte für zu niedrig.

Die häufigere Manifestation der Erkrankung bei dem inkretorisch stärker beanspruchten weiblichen Geschlecht darf als gesichert angesehen werden. Gowers, Moebius und Curschmann fanden eine Relation von Frauen zu Männern wie 1,5 zu 1. Die Verhältniszahl von E. Flateau betrug 2,7 zu 1; nach Bing kommt auf 4 Manifestationen bei Frauen ein kranker Mann. H. Richter fand bei Patienten einer Poliklinik ebenfalls das Verhältnis 4 zu 1, bei Privatpatienten dagegen nur 1,7 zu 1. Der Untersucher erklärt diesen Unterschied mit einer Annahme Curschmanns, nach der sich die Migräne bei intellektuellen Berufen entschieden häufiger manifestiert. Richter zitiert Befunde von Allan, nach denen die Erkrankungszahlen in beiden Geschlechtern gleichgross seien und auch die vorherrschende Meinung einer bevorzugten Vererbung von der Mutter auf die Tochter nicht richtig sei. Die Annahme einer bevorzugten Vererbung anfallartiger Kopfschmerzen durch das weibliche Geschlecht geht vor allem auf frühere Beobachtungen von E. Flateau zurück. 
Zahlreiche Familienuntersuchungen, vor allem von Ball, Moloney, R. M. Balyeat, H. J. Rinkel, Gowin, Rove u. a., sprechen für das Vorkommen einer ungewöhnlich hohen Zahl allergischer Manifestationen in den Familien der Hemikraniekranken. Aus den Prozentzahlen, die diesen Mitteilungen beigefügt sind, ist jedoch häufig nicht zu entnehmen, welcher Personenkreis im einzelnen Falle zur Familie des Probanden gerechnet wurde. Deshalb sind die Ziffern als Vergleichsmaterial nicht immer brauchbar.

Nach Berger lässt sich eine allergische Diathese beim Menschen auf nur wenige Grundfunktionen zurückführen. Diese Regulationsanomalien beruhen auf einer veränderten Reaktibilität bei Tonusverschiebungen im autonomen Nervensystem. "Welche Vorgänge im Stoffwechsel die Bereitschaft zur Migräne gewährleisten, ist unbekannt » (H. Pette). Der für die pathogenetische Betrachtung der Hemikranie "wesentliche Schnittpunkt " sei das Vorkommen gleicher Syndrome « in buntem Durch- und Nebeneinander" in Familien mit Belastung durch Migräne oder Allergie. Was vererbt werde, sei somit weder die Anlage zu der einen oder der anderen Krankheit, sondern « eine äusserst vasolabile Konstitution ».

Laesionen der vegetativen Peripherie, wie endokrine Dysregulationen (" ovarielle Migräne »), cerebrale Durchblutungsstörungen (P. Sunder-Plassmann u. Mitarb.), Mikroventrikulie (F. A. Kehrer), Missbildungen des Schädelskeletts (wie Turricephalie), pathologische Veränderungen im Bereich der Halswirbelsäule ("migraine cervicale ", W. Bärtschi-Rochaix), psychische Reaktionen (A. R. Furmanski) können bei genuiner Krampfbereitschaft auslösende Reize darstellen. H. Richter beobachtete die Heilung einer gleichseitigen Hemikranie nach Entfernung von Geschwülsten im oberen Thoraxbereich. Die gleichen Bedingungen sind bei iktophiler Konstellation nach G. Koch geeignet, einen epileptischen Krampfanfall auszulösen.

Das gleichzeitige oder alternierende Vorkommen von Migräne und Epilepsie ist in vielen Sippen und bei vielen Einzelindividuen beschrieben worden (Revington, Liveing, Flateau, Richter, Kehrer, Klimke u. a.). Epilepsie und Migräne bei einer Person sah M. Ulrich in 3,4\% und $\mathrm{H}$. Richter bei 3,4\% seiner Migränekranken. Nach F. A. Ely litten von 171 Epileptikern 15\% auch an Migräne und von 104 Migränekranken 8,6\% auch an Epilepsie. Nach einem Vortrag von H. Hoff aus dem Jahre 1954 gehören migräneartige Anfälle als atypische Manifestationen zum Bilde der Epilepsie. Damit ergibt sich die Frage, ob diese Form der Hemikranie dem " Grenzgebiet der Epilepsie " (O. Foerster) zuzurechnen ist.

Zwillingsuntersuchungen ermöglichen eine Unterscheidung zwischen der Wirkung von Erbe und Umwelt, den beiden Kräftegruppen der Entwicklung. D. Spaich und M. Ostertag fanden bei 10 erbgleichen Zwillingspaaren in allen Fällen eine Konkordanz hinsichtlich allergischer Manifestationen, aber nur in 6 Fällen in bezug auf Migräne. Fünf zweieiige Paare zeigten viermal Konkordanz bei der Allergie, jedoch nur zweimal in der Manifestation von Migräne. Weitere Zwillingsbeobachtungen veröffentlichten M. Ulrich (PZ, konkordant bzgl. Migräne), Trousseau (EZ ?, konkordant), Siemens (1 EZ, diskordant und $1 \mathrm{EZ}$ ? konkordant), Matzdorff (EZ, konkordant), Schulte EZ, konkordant). Die Zwillinge von Matzdorff und Schulte zeigten eine Manifestation der Migräne in verschiedenem Lebensalter. Kahler und Weber beobachteten konkordante 
Hemikranie bei $2 \mathrm{EZ}$ und $1 \mathrm{ZZ}$, Diskordanz bei 6 erbverschiedenen Paaren. BorupSvendsen hat 1952 den « different course of migraine in monocygotic twins " beschrieben. Obwohl es sich bei diesen $30 \mathrm{Zwillingspaaren} \mathrm{der} \mathrm{Literatur} \mathrm{keineswegs} \mathrm{um} \mathrm{eine} \mathrm{auslese-}$ freie Serie handelt, dürfte die Uebersicht einen ersten Eindruck vermitteln: Von den 16 sicher erbgleichen Paaren zeigten 12 Paare Konkordanz in bezug auf die Migränemanifestation; von den 13 erbverschiedenen Zwillingen waren dagegen nur 4 Paare konkordant. Bei diesen Werten ist allerdings zu beachten, dass die Konkordanzziffer bei der Summe der veröffentlichten Einzelfälle sicher höher liegt als den tatsächlichen Verhältnissen entspricht. Ausserdem dürfte die Differentialdiagnose u. a. zwischen häufiger auftretenden einfachen Kopfschmerzen und der typischen Migräne, jedenfalls in Grenzfällen, nicht bei allen Untersuchern völlig übereinstimmend gestellt worden sein. Trotzdem scheint das Ergebnis der bisherigen Zwillingsstudien eine frühere Folgerung von P. Matzdorff zu bestätigen, nach der die Migräne eine erbbedingte Krankheit ist, deren Manifestation eine weitere anfallauslösende Ursache erfordert.

\section{Eigene Untersuchungen}

Dank der systematischen Befragung aller Patienten der Psychiatrischen und Nervenklinik der Universität Münster, die der ehemalige Direktor der Klinik, Herr Professor Dr. F. A. Kehrer, seit dem Jahre 1936 vornehmen liess, und die von dem jetzigen Direktor, Herrn Prof. Dr. F. Mauz, fortgeführt wird, konnten wir eine auslesefreie Zwillingsserie für die vorliegende Untersuchung ermitteln. In den Jahren vom 1-1-1936 bis 31-12-1953 sind insgesamt 30259 Patienten wegen neurologischer und psychiatrischer Krankheiten stationär behandelt worden. Darunter waren $19 \mathrm{Zwillingspaare,} \mathrm{von} \mathrm{wel-}$ chen jeweils ein Partner klinisch beobachtet worden ist. ${ }^{3}$ Es handelt sich um 10 weibliche, 3 männliche und 6 Pärchenzwillingspaare. Von den $6 \mathrm{PZ}$ ist in vier Fällen der weibliche und zweimal der männliche Partner behandelt worden. Somit ergibt sich insgesamt ein starkes Ueberwiegen der weiblichen Ausgangsprobanden im Verhältnis 14:5. Die Zwillingsdiagnose war bei 13 Paaren möglich; unter diesen sind 3 (weibliche) EZ, 5 (weibliche) ZZ und 5 PZ. Bei den restlichen 6 Zwillingspaaren konnte eine Aehnlichkeitsdiagnose bisher nicht durchgeführt werden.

Diese vorläufige Mitteilung beschränkt sich im wesentlichen auf das Ergebnis unserer Untersuchungen bei den 13 Paaren mit gesicherter Zwillingsdiagnose. In einer späteren Darstellung werden neben den restlichen 6 Paaren auch die gleichzeitig erhobenen Familienbefunde, bei einzelnen Paaren abgeleitete Elektroencephalogramme und die Kasuistik, ausführliche Berücksichtigung finden. Einen Ueberblick über alle 13 Paare gibt Tabelle 1:

${ }^{3}$ Herrn Prof. Dr. F. Mauz, dem jetzigen Direktor der Klinik, sind wir für die Mitteilung der Namen und Anschriften der Patienten zu Dank verpflichtet. 
Tab. I - Uebersicht der Gesamtserie von ${ }_{3} 3$ Zwillingspaaren

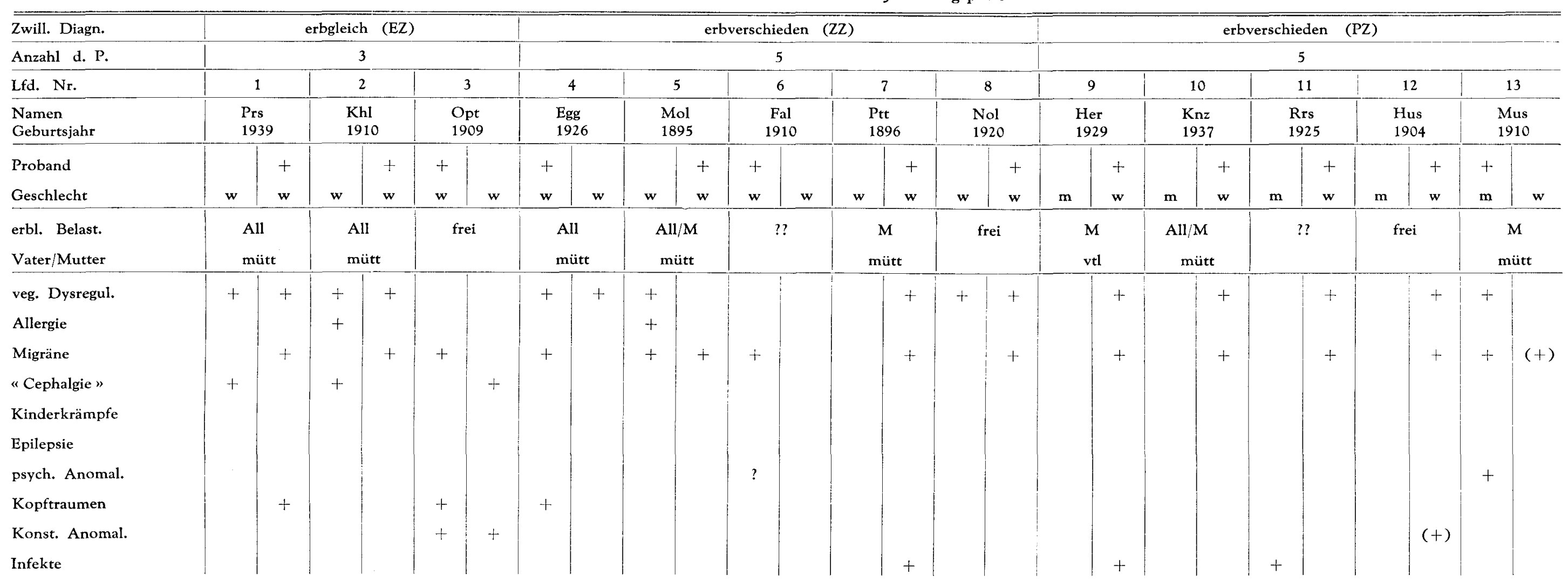

Zeichenerklärung: All $=$ Allergie, $\quad M=$ Migräne; das Symbol + gibt den Probanden oder das Vorliegen der betr. Krankheit an 


\section{Erbgleiche Zwillinge}

Die drei eineiigen Zwillingspaare sind sämtlich weiblichen Geschlechts. Paar 3 unterscheidet sich wegen einer konkordanten Turricephalie von den Zwillingen 1 und 2, die ihrerseits durch Vorkommen von allergischen Manifestationen bei den Müttern und, im Falle 2, bei einem Sohn der erstgeborenen Schwester belastet sind.

Das erste Zwillingspaar erlebte ein Flüchtlingsschicksal und den Kriegstod des Vaters, eines Automechanikers. Infolge der Vertreibung kamen die jetzt 16-jährigen Mädchen zwei Jahre später auf die Oberschule. I ist realistischer, mehr praktisch veranlagt und möchte Gewerbelehrerin werden, während die zweitgeborene Probandin ein Medizinstudium anstrebt, für das eigentlich die wirtschaftlichen Voraussetzungen fehlen. Beide Schwestern sind gute, ehrgeizige Schülerinnen. II leidet seit dem 14. Lebensjahr unter Migräneanfällen, die sich besonders bei Unregelmässigkeiten der Lebensführung im Sinne einer "Sonntagsmigräne" manifestieren. I hat nach ihren eigenen Angaben und der Mitteilung der Mutter nicht unter diesen typischen Anfällen, dagegen aber unter häufigen Kopfschmerzen, besonders zur Zeit der Menstruation und bei Witterungswechsel zu klagen. Die $Z$ willinge 1 sind, wie die Mutter, vegetativ stark dysreguliert.

Unsere zweiten Zwillingsschwestern, 45 Jahre alt, entstammen einer Beamtenfamilie und sind mit Verwaltungsangestellten verheiratet. Die Zweitgeborene wird von I als " etwas eitel und sehr wählerisch " gekennzeichnet, während sie selbst immer das "Arbeitstier» gewesen sei. II habe mehr an der Mutter gehangen. Beide Sschwestern wurden mit 14 Jahren zu verschiedenen Meisterinnen in die Schneiderlehre gegeben. Während nun I die Lehre mit Erfolg beendet hat und schon in jungen Jahren ein eigenes Geschäft gründete, versagte II sehr bald in der Lehre, blieb als Haustochter bei den Eltern und betätigte sich später als "Empfangsdame " bei der Schwester. I heiratete und ist Mutter von 3 Kindern. Neben ihrer Tätigkeit als Hausfrau und Mutter versorgt sie einige Untermieter in einem eigens dafür gepachteten grossen Haus. II blieb bis zum Tode der Eltern vor zwei Jahren bei der Mutter und heiratete dann als dessen zweite Frau einen Grubenangestellten. In ihren jungen Jahren habe sie nie einen passenden Ehepartner gefunden. Während I sehr unruhig und geschäftig ist, wegen ihrer «inneren Unruhe » Sedativa verordnet bekommt, leidet II seit ihren Jungmädchenjahren unter schwerem Migränekopfschmerz, der seit der Eheschliessung allerdings an Intensität verloren hat. Interkurrierend infizierte sie sich luetisch und wurde wegen einer L 2 behandelt.

Bei dem 46-jährigen 3. Paar handelt es sich um schlesische Kleinbauerntöchter. Unsere Probandin, I, arbeitete auf dem Hof der Eltern, nach der Vertreibung als Industriearbeiterin. II heiratetet einen Waldarbeiter. Wie erwähnt, haben beide Schwestern eine Turricephalie. Schon vor wiederholten Kopftraumen bei Kriegsende litt I an anfallartigen Kopfschmezen, besonders nach Sonneneinwirkung bei der Feldarbeit, aber auch nach Schlafentzug und ähnlichen Belastungen. II leidet häufiger unter leichteren Kopfschmerzen, die jedoch nie anfallartig auftreten; auch sie fühlt sich nach sommerlicher Feldarbeit unwohl und neigt zu Erbrechen dabei.

Zusammenfassend lässt sich bei unseren EZ also sagen, dass sie in bezug auf typische Migräneanfälle sämtlich diskordant sind. Hinsichtlich Cephalgien überhaupt besteht 
eine Konkordanz. $\mathrm{Ob}$ es sich dabei um Manifestationsschwankungen der Migräneanlage handelt, oder aber um das oft beobachtete Gleichsein-wollen der Zwillinge, lässt sich schwer sagen. Auffallend ist jedoch die Konkordanz in der vegetativen Labilität bei allen Paaren. Ein weiterer Hinweis für die Annahme von Manifestationsschwankungen dürfte in der besonderen psychischen Situation der jeweils erkrankten Partner gegeben sein. Bei den Paaren 1 und 2 wird es sich im Hinblick auf die allergische Belastung durch die Mütter und das Fehlen anderer neurologischer oder interner Abweichungen um eine "genuine" Migräne handeln. Wegen der Turricephalie und des Fehlens anderer Anomalien ist die Migräne beim Paar 3 vermutlich ein "symptomatisches" Krankheitsbild mit Manifestationsschwankungen auf dem Boden genuiner Krampfbereitschaft.

\section{Erbverschiedene Zwillinge}

Von den insgesamt 5 weiblichen zweieiigen Zwillingspaaren lässt sich das Paar 6 abgrenzen: II ist hinsichtlich unserer Fragestellung völlig gesund, I zeigt neben dem Auftreten anfallartiger vasomotorischer Kopfschmerzen eine vegetative Symptomatik. Bemerkenswert ist, dass die Probandin psychische Anomalien zeigt, die als Ursache oder Folge schwerer Ehekonflikte mit anschliessender Scheidung anzusehen sind. Der Manifestationstermin der Hemikranie liess sich nicht genau ermitteln, wahrscheinlich bestand jedoch schon vor der Heirat eine Migräne. Die Paare 4, 5 und 7 sind von der Mutter her mit Allergie belastet; bei den Zwillingen 5 soll schon die Mutter unter anfallartigen Kopfschmerzen gelitten haben. Beim Paar 8 konnte Erblichkeit nicht nachgewiesen werden.

Lediglich beim Paar 5 besteht eine Konkordanz hinsichtlich der Migräne. Die Erkrankung hat sich am stärksten bei II manifestiert. Besonderer Beachtung bedarf auch der

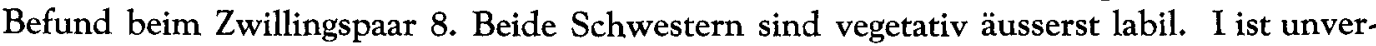
heiratet und Sprechstundenhelferin bei einem Ophthalmologen. Bei der Probandin II traten erstmals mit 20 Jahren anfallartige Kopfschmerzen auf. Sie leidet unter einer Dysmenorrhoe. Infolge unglücklicher ehelicher Verhältnisse scheint eine Verstärkung der Hemikranie eingetreten zu sein. Beim Paar 4, jetzt 28-jährigen Büroangestellten, liegt eine konkordante vegetative Dysregulation mit allgemeiner psychischer Unausgeglichenheit vor. Ein leichtes Kopftrauma bei der Probandin dürfte pathogenetisch kaum von Bedeutung oder aber lediglich auslösend wirksam geworden sein; es ist eher anzunehmen, dass es Anlass zur Lösung einer unbefriedigenden Anstellung gewesen ist. Der unerfüllte Wunsch nach Verbesserung der äusseren gesellschaftlichen Stellung dürfte bei beiden Schwestern pathogenetisch bedeutungsvoll sein. Die Migräne bei I manifestiert sich etwa seit dem 16. Lebensjahr; ungefähr in den letzten acht Jahren ist eine Verstärkung eingetreten. Die Migräne tritt vorzüglich nach der Art der «Sonntagsmigräne " auf.

Von den $5 \mathrm{PZ}$ sind 3 Paare auch erblich belastet, 9, 10 und 13. Beim Paar 11 konnten wir entsprechende Mitteilungen noch nicht sichern. Als schwach konkordant ist lediglich das Zwillingspaar 13 anzusprechen, alle anderen Paare verhalten sich diskordant.

Besonderer Besprechung bedarf das schwach konkordante Zwillingspaar 13. Die 
Mutter und deren Schwester litten unter schweren anfallartigen Kopschmerzen. Die Zwillingsschwester II leidet während der Menstruation und bei Witterungswechsel unter anfallartigen, aber "nicht so häufig» auftretenden Kopfschmerzen. Der Proband I leidet unter anfallartigen Depressionszuständen, die anscheinend als Aequivalent möglicherweise aber auch reaktiv nach einem Migräneanfall auftreten. Der Zwillingsbruder der Probandin (II) aus dem Paare 11 starb angeblich an "Keuchhusten". Manche anamnestischen Angaben lassen es jedoch wahrscheinlicher erscheinen, dass es sich dabei um Kinderkrämpfe gehandelt hat. Bemerkenswert ist die schwere allergische Belastung in der engeren Familie des Paares 10, bei dem nur der weibliche Partner erkrankt ist. Bei der Zwillingsschwester von 12 traten schwerste Migräneanfälle zum ersten Male nach einer Exstirpation der Ovarien auf. Sowohl der Zwillingsbruder als auch die übrige Verwandtschaft sind völlig frei von einer in diesem Rahmen wichtigen Krankheit.

Wenn wir die Beobachtungen bei den insgesamt 10 erbverschiedenen Paaren zusammenfassen, so dürften 3 Paare an "symptomatischen » Manifestationen leiden $(6,11,12)$, in den übrigen Fällen handelt es sich vermutlich um "genuine " Erkrankungen. Insgesamt findet sich bei den $\mathrm{ZZ}$ und den $\mathrm{PZ}$ nur einmal eine Konkordanz, Nr. 5 (ZZ), und bei einem weiteren Paar eine schwache Konkordanz, Nr. 13 (PZ). In beiden Fällen handelt es sich um "genuine " Migräneformen bei erblicher Belastung. Hinsichtlich vegetativer Dystonie sind nur zwei ZZ (Nr. 4 und 8) konkordant.

\section{Ergebnisse}

Tab. 2 - Migräne

\begin{tabular}{c|c|c|c|c}
\hline & $\mathrm{k}$ & $\mathrm{kd}$ & $\mathrm{d}$ & $\Sigma$ \\
\hline $\mathrm{EZ}$ & 0 & 3 & 0 & 3 \\
$\mathrm{ZZ}$ & 1 & 1 & 3 & 5 \\
$\mathrm{PZ}$ & 0 & 1 & 4 & 5 \\
\hline$\Sigma$ & 1 & 5 & 7 & 13 \\
$4=$ & gleichgeschlechtliche Paare der Gruppe 2.
\end{tabular}

Tabelle 2 gibt einen Ueberblick unserer vorläufigen Serie von 13 Zwillingspaaren. Als konkordant (k) wurden nur die Paare angesehen, in denen sich eine typische Migräne bei beiden Partnern manifestierte. Im Laufe der Untersuchung hat sich oft herausgestellt, dass der Partner der migränekranken Probandin unter häufigen Kopfschmerzen litt, welche jedoch nicht die typische Symptomatologie der Hemikranie boten. Diese Patienten wurden deshalb zu einer besonderen Gruppe zusammengefasst und als konkordant-diskordant (kd) bezeichnet.

Es ist erstaunlich, dass sämtliche EZ-Paare unserer Serie in dieser Weise als konkordant-diskordant anzusprechen waren. Bei den ZZ-Paaren betrug das Verhältnis $\mathbf{k}: \mathbf{k d}$ : $\mathrm{d}=1: 1: 3$ und bei den Pärchenzwillingen sogar nur $0: 1: 4$. Insgesamt zeigt sich also, dass die Relation $\mathrm{k}+\mathrm{kd}$ zu d bei den EZ $3: 0$, bei den $\mathrm{ZZ} 2: 3$, bei den PZ1 : 4 und den erbverschiedenen Zwillingspaaren insgesamt ( $Z Z+P Z) 3: 7$ ausmachte. Mit anderen Worten: alle unsere EZ-Paare sind in dieser Weise als konkordant anzusprechen, von den erbverschiedenen Zwillingspaaren sind 3 konkordant und 7 diskordant.

Die Berechtigung der Zusammenfassung der $k$ - und kd-Gruppen ergibt sich aus dem 
Befund der Tabelle 3. Obwohl leichte und mittlere vegetative Dysregulationen bei einem sehr hohen Pronzentanteil der Bevölkerung bestehen, waren die Befunde bei unseren Zwillingen jedoch so auffallend, dass sie über die Erwartung hinausgingen. Eine vegetative Dysregulation wurde von uns dann diagnostiziert, wenn neben subjektiven Symptomen Dermographismus, feuchte und kalte

Tab. 3 - Vegetative Dysregulation

\begin{tabular}{c|c|c|c}
\hline \hline & $\mathbf{k}$ & $\mathrm{d}$ & $\Sigma$ \\
\hline $\mathrm{EZ}$ & 3 & 0 & 3 \\
$\mathrm{ZZ}^{4}$ & 2 & 3 & 5 \\
$\mathrm{PZ}$ & 0 & 5 & 5 \\
\hline$\Sigma$ & 5 & 8 & 13
\end{tabular}

Hände, respiratorische Arhythmie, nervöse Uebererregbarkeit, Schlafstörungen, und verwandte Symptome beobachtet werden konnten. Ein weiterer Anhalt für die Richtigkeit unseres Vorgehens ergibt sich aus der Tabelle 3 selbst.

Hinsichtlich einer vegetativen Dysregulation waren alle unsere EZ-Paare konkordant. Bei allen erbverschiedenen Zwillingen zusammen fanden wir eine $\mathrm{k}: \mathrm{d}$-Relation von $2: 8$. Somit dürfte ein Erbfaktor, soweit die kleinen Zahlen dieses Urteil erlauben, bei der Manifestation der vegetativen Dysregulation wirksam sein. Diese Annahme wird bestätigt durch die Untersuchungen von Kahler und Weber, die "bei erbgleichen Zwillingen regelmässig konkordantes Verhalten von Zeichen vegetativer Uebererregbarkeit " (zit. H. Losse) gefunden haben und auf " die hervorragende Bedeutung der Erbveranlagung für diese Zustandsbilder" hinweisen konnten. Zu gleichen Ergebnissen kommt $H$. Losse in einer kürzlich abgeschlossenen Untersuchungsreihe. Die unterschiedliche Konkordanzziffer erlaubt ausserdem ein Urteil über die Richtigkeit der beschriebenen Addition der $k$ - und kd-Werte in Tabelle 2. Nur ein ZZ-Paar und ein PZ-Paar zeigen, bei Konkordanz hinsichtlich Kopfschmerzen, nicht gleichzeitig beide eine vegetative Dystonie. Nach einem Vergleich mit Tabelle 1 handelt es sich dabei um die Paare 5 und 13, bei denen uns jedoch jeweils weitere Migränefälle in der Familie bekannt geworden sind. Umgekehrt leiden von den Paaren 4 und 8 trotz erblicher Belastung und Konkordanz hinsichtlich vegetativer Dystonie nur die Probanden an Migräne, ein Befund, der im folgenden weitere Besprechung erfahren wird. Die unterschiedlichen Konkordanzziffern bieten ausserdem einen Hinweis auf die Richtigkeit der Annahme einer erhöhten Zahl ausgeprägt vegetativ Dystoner bei Migräne gegenüber der allgemeinen Erwartung in der Bevölkerung.

In der Tabelle 4 sind wir der Frage der erblichen Belastung unter dem Blickwinkel der Konkordanz oder Diskordanz für Migräne nachgegangen. Der deutlichste Befund ist bei den EZ gegeben. Während von den 3 bezüglich Cephalgien überhaupt konkordanten EZ-Paaren 2 eine entsprechende Manifestation in der Elterngeneration aufweisen, konnte bei einer Befragung des dritten Paares diese Beobachtung auch in der Generation der Grosseltern nicht gemacht werden. Gerade bei diesem EZ-Paar Nr. 3 waren wir unsicher bei der Einordnung in die $k$ - oder kd-Gruppe, weil die Beschwerden der Partnerin doch in vieler Beziehung Aehnlichkeit haben mit typischer Migräne. Bei den anderen beiden EZ-Paaren liegt wohl eine erbliche Belastung hinsichtlich einer Allergie, aber nicht in bezug auf Migräne vor. Diese Beobachtungen sind bemerkenswert und 
zeigen hin auf die konstitutionellen Vorbedingungen und die Erbverhältnisse im Migränekreis überhaupt. Bei den erbverschiedenen Zwillingen waren zwei Paare mit Migräne und Allergie gleichzeitig erblich belastet (Nr. 5 und Nr. 10). Zwei weitere Paare (Nr. 6 und Nr. 11) mussten wir fortlassen, weil die Daten der Familienanamnese noch nicht

Tab. 4 - Erbliche Belastung in den Generationen der Eltern und Grosseltern unserer Zwillinge ${ }^{5}$

\begin{tabular}{l|c|c|c|c|c}
\hline \hline & $\begin{array}{c}\mathrm{EZ} \\
\mathbf{k}+\mathbf{k d}\end{array}$ & $\begin{array}{c}\mathrm{EZ} \\
\mathbf{d}\end{array}$ & $\begin{array}{c}\mathrm{ZZ} \\
\mathbf{k}+\mathrm{kd}\end{array}$ & $\begin{array}{c}\mathrm{ZZ} \\
\mathrm{d}\end{array}$ & $\Sigma$ \\
\hline Allergie & 2 & 0 & $(1)$ & $1+(1)$ & 3 \\
Migräne & 0 & 0 & 2 & 3 & 5 \\
frei & 1 & 0 & 0 & 2 & 3 \\
\hline \multicolumn{1}{c|}{} & 3 & 0 & 2 & 6 & 11
\end{tabular}

5 zwei diskordante ZZ-Paare unsicherer erblicher Belastung wurden nicht verwendet.

gesichert werden konnten. Eine erbliche Belastung für Migräne oder Allergie konnte bei den ZZ-Paaren 8 und 12 nicht gefunden werden. Wie aus der Kasuistik hervorgeht, leidet die Probandin des Paares 12 (PZ) an einer schweren ovariellen Migräne nach Exstirpation der Ovarien. Die Zwillingsschwestern 8 sind zwar diskordant bezüglich Migräne, aber konkordant hinsichtlich einer vegetativen Dysregulation. Die Probandin ist wegen einer Dysmenorrhoe behandelt worden, ein gynäkologischer Befund steht noch aus. Es ist vorstellbar, dass in beiden Fällen (12 und 8) eine endokrine Störung massgeblich an der Migränemanifestation beteiligt ist.

Die erbliche Grundlage der Migräne dürfte in einer Bereitschaft zu gesteigerter vegetativer Reaktion bestehen. Ob sich diese genetische Grundlage als vegetative Dystonie, Migräne, Allergie oder sonstwie klinisch manifestiert, liegt in der spezifischen Reaktibilität des Einzelorganismus auf zusätzliche endogene oder exogene praeformierende Reize begründet. Mit anderen Worten: Die Manifestation einer Migräneanlage ist abhängig von vielfältigen modifizierenden Einflüssen. Es sind zwei Faktoren (P. Matzdorff) erforderlich, eine erbliche Teilursache und sehr heterogene andere Bedingungen. Die Unterteilung der Migräne in genuine und symptomatische Formen ist nosologisch daher wahrscheinlich nicht berechtigt. Bei hoher Anfallbereitschaft genügen schon geringe Reize und umgekehrt. Weil die genetische Ursache wirksam wird bei einem vielfältigen Regulationssystem, kann die, nach Familienuntersuchungen anderer Autoren, dominant erbliche Anlage auch aus diesem Grunde bei ausreichender Kompensation latent bleiben. Das Problem Migräne und Epilepsie klammern wir in dieser vorläufigen Mitteilung zunächst aus.

Die häufig diskutierte Frage der bevorzugten «Vererbung" von der Mutter auf die 
Tochter und die häufigere Manifestation beim weiblichen Geschlecht - beides konnten auch wir beobachten - findet so eine Erklärung. Der weibliche Organismus ist vegetativhormonal stärker beansprucht als der des anderen Geschlechts. Das Fehlen einer Kompensationsreserve führt daher bei Frauen leichter zu einer Manifestation.

Die Bedeutung des Geistig-Seelischen (Furmanski) für die Manifestation der Migräne oder das stärkere Auftreten der Hemikranie bei einem Paarling wird besonders deutlich bei den EZ 1 und 2. Bei 1 und 2 standen die stärker erkrankten Probandinnen jeweils in einer Konfliktsituation. Die Beobachtung von H. Richter und Curschmann, nach der sich die Hemikranie bei intellektuellen Männern häufiger manifestiert, mag Ausdruck der stärkeren geistigen Beanspruchung und der erhöhten Konfliktgefährdung sein.

Die erbliche Belastung mit allergischen Diathesen bei Fehlen einer entsprechenden Migränemanifestation $\mathbf{u}$. a. in den Familien unserer EZ-Paare Nr. 1 und Nr. 2 deutet darauf hin, dass Allergie und Migräne zueinander in pathogenetischer Beziehung stehen. Nach E. Hanhart ist die vegetative, diencephale Regulation beim Allergiekranken selten gestört. Besteht jedoch gleichzeitig mit der anaphylaktischen Reaktionsfähigkeit eine vegetative Dystonie, wird eine allergische Diathese verstärkt. $H$. Pette definiert die Migräne als "ein praeformiertes Geschehen» und "als Entäusserung einer vegetativen Dystonie ». Es wird also sicher so sein, das bestätigen auch unsere Befunde bei Zwillingen, dass eine vegetative Dysregulation sowohl die Allergie als auch einen Migräneanfall verstärken, bzw. die latente Manifestation erst auslösen kann, i. e. dass die primär vorhandene vegetative Dystonie auf diesem oder jenem praeformierten Wege zur Entladung kommt. Dafür spricht auch die konkordante vegetative Dystonie bei unseren EZ-Paaren.

Die theoretische Aufschlüsselung verliert sich freilich wegen der lokalisatorischen Beziehungen der Zentren im Zwischenhirn und wegen der dauernden Wechselwirkung zwischen vegetativer Peripherie und Zentrale bei der personalen Krankheit. Es erscheint uns jedoch wichtig, herauszustellen, dass der Migräne eine erbliche diencephale Funktionsschwäche zugrunde liegt, "bei der einfach alles in Mitleidenschaft gezogen werden kann, was vom Zwischenhirn aus reguliert wird (Kibler)".

\section{Zusammenfassung}

Es wird über eine auslesefreie Serie von 19 Migränezwillingen berichtet. Die Befunde bei insgesamt 13 Paaren mit gesicherter Zwillingsdiagnose, 3 erbgleiche und 10 erbverschiedene, werden vorläufig zusammengefasst. Konkordanz hinsichtlich typischer Migräne zeigte nur ein erbverschiedenes Paar; eine schwache Konkordanz wurde bei allen EZ und $3 \mathrm{ZZ}$ gefunden. Bezüglich einer ausgesprochenen vegetativen Dystonie waren dagegen sämtliche erbgleichen und zwei erbverschiedene Paare konkordant. Nach diesen Ergebnissen sind an der Manifestation einer Hemikranie verschiedene Teilursachen beteiligt. Die erbliche Grundlage scheint in einer vegetativen, diencephalen Dysregulation gegeben zu sein. Die klinische Form der Entäusserung wird durch weitere, sehr unterschiedliche, endogene und exogene praeformierende Einflüsse bestimmt. 


\section{Literatur}

Allan, W.: Arch. Int. Med. 42 (1928, 590, ders.: Arch. Neurol. (Am.). 18 (1927), 507.

BaErTschi, W.-Rochaix: 13. Congr. Soc. Franc. Oto-Neuro-Ophthalm., Strassbourg 1946, ref. Zbl. Neurol., 108 (1950), 259, ders.: Praxis 1948, 673-677, ders.: Praxis 1953, 966-967.

Ball, F. E.: Amer. I. Med. Sci. 173, 1927 cit. Richter H., Hb. d. Neurol.

Balyeat, R. M.: Genetics 18 (1933), 134, ders, und Brittain F. L.: Am. J. Med. Sci. 180 (1930) 212. Berger: cit. EICHLER, L.-OHEIM, Fortschr. Erbpathol. 7-8 (1943-44), 1-29.

Bing, R.: Lehrb. d. Nervenkrankh., cit. Richter, H., Hb. d. Neurol.

Bourup, B.-Svendson: Acta psychiat. neurol. Scand. 12:27 (1952), 165.

Curschmann, H.: Med. Welt 1117-1121, 1940, ders.: Münch. med. Wschr. I 1924, 111, ders.: Nervenarzt 4 (1931), 71 .

CURtiUs, F.: Die organischen und funktionellen Erbkrankheiten des Nervensystems, Stuttgart, 1935. EICHLER, L.-OHEIM: Fortscr. Erbpathol., 7-8 (1943-44), 1.29.

Eley, F. A.: Arch. Neurol. (Am.) 24 (1930), 943-944.

Flateau, E.: Die Migräne, Berlin, 1912, cit. Richter, H., Hb. d. Neurol.

Furmanski, A. R.: Arch. Neurol. (Am.), 67 (1952), 23-31.

Girard J. und Colleson, L.: Presse méd, I 1939, 707.

Gowers: Brit. Med. Journ. 1906, ders. : Diseases of the Nervous System, London 1883 cit. RrCHTER, H., $\mathrm{Hb}$. Neurol.

GRUNNERT, K.: Münch. med. Wschr. I 1939, 841-847, ders.: Münch. med. Wschr. II 1938, 1337-1341.

HanharT, E.: Schw. med. Wschr. 12:71 (1941), 465, ders.: Dtsch. med. Wschr. 29-31 (1934), 1110. Hess, R. W.: cit. KibleR, M.: Med. Welt 27-28 (1951), 881.

HeYerdaHL: cit. EICHLER, L.- OHEIM, Fortschr. Erbpathol. 7-8 (1943-44).

Hoff, H.: Wien. klin. Wschr. 8:67 (1955), 137-140.

JANSEN, R.: Dtsch. Z. Nervenheilk. 155 (1943), 42-68, ders.: Münch. med. Wschr. 15-97 (1955), 463466.

KaHLeR, O. H. und Weber, R.: Zschr. klin. Med. 137 (1940) 380-477 \& 507-575.

KEHRER, F. A.: Dtsch. Z. Nervenheilk. 163 (1950), 555.

KiBLER, M.: Med. Welt 27-28 (1951), 881.

KLIMKE, W.: Therapiewoche 11-12: III (1953), ders. Z. Neurol. Psychiat. 131 (1933), 542.

Kock, G.: Die Krampfbereitschaft, Edizioni dell'Istituto Gregorio Mendel, Roma 1955.

LiveING: cit. EichleR, L.-OHEIM, Fortschr. Erbpathol. (1943-44).

Losse, H.: Die vegetative Struktur des Individuums, Habil. Schrift (Manuskript), Münster 1955.

MatzdorfF, P.: Z. Neurol. 156 (1936), 574, ders.: Dtsch. Z. Nervenheilk. 146 (1938), 76-101, ders.: Mschr. Unfallheilk. 44 (1937), 600-616, ders.: Nervenarzt 12 (1939), 225-239, ders.: Nervenarzt 13 (1940), 529-537, ders.: Med. Klin. II (1940), 1434-1436, ders.: Klin. Wschr. II (1940), 720, ders.: Nervenarzt 15 (1942), 204-208.

MaUz, F.: Zbl. Neurol. 45, 833-835 (1927), ders. Dtsch. med. Wschr. 55, 825-826 (1930).

Moebius: Die Migräne, Wien 1894 cit. EICHLER, L.-OHeim, Fortschr. Erbpathol. 1943-44.

MoEHLig, R. C.: Endocrinology (Am.) 15 (1931), 11.

Mendel, E.: Disch. Med. Wschr. I (1906), 725 cit. Eichler, L.-Oheim, Fortschr. Erbpathol. 1943-44. Moloney, J. C.: Arch. Neurol. (Am.) 19 (1928), 684 cit. Richter, H., Hb. d. Neurol.

Pette, H.: Dtsch, med. Wschr. 15:80 (1955), 523-525.

Richter, H.: Die Migräne; in Bumke-Foerster, Hb. d. Neurol., XVII, 166-245, Springer, Berlin 1935. RINKEL, H. J.: J. Allergy (Am.) 4 (1933), 303.

Rove, A. H.: California Med. 33 (1930), 785 cit. Richter, H., Hb. d. Neurol.

Schulte, H.: Berl. Ges. Psychiat. Nervenheilk. 1930, ref. Zbl. Neurol. 59 (1931), 266.

Siemens, H.W.: cit. Eichler, L.-Oheim, Fortschr. Erbpathol. 1943-44.

Spaich, D. und Ostertag, M.: Z. menschl. Vererbungsl. 19 (1936), 73.

Sunder, Plassmann, P., Hillenbrand, H. J. und Schurholz, A.: Münch. med. Wschr. 96 (1954), 
Trousseau: cit. Erchler,L.-Oheim, Fortschr. Erbpathol. 1943-44.

Ulrich, M.: Mschr. Psychiat. Neurol. 31 (1912), 134 cit. Richter, H., Hb. d. Neurol. v. Verschuer, O.: Erbpathologie, 2. Aufl., Steinkopff, Dresden \& Leipzig 1937.

WolfF, H. G.: Triangel (Sandoz) 2:2 (1955), 53.

Zainden: cit. HirschmanN, J., Therapiewoche 21-22:4 (1954), 537.

\section{RIASSUNTO}

Viene riferito in merito ad una casistica relativa a 19 casi di gemelli affetti da emicrania ed ai rilievi eseguiti su 13 coppie, la cui diagnosi di gemellanza (3 $M Z$ e Io DZ) era stata accertata.

Riguardo all'emicrania tipica fu mostrata concordanza soltanto da I coppia $\mathrm{DZ}$; una debole concordanza fu trovata in tutte le coppie $M Z$ ed in tre $\mathrm{DZ}$. In riferimento però ad una pronunciata distonia vegetativa si mostrarono concordanti tutte le coppie $M Z$ e due DZ. Secondo tali risutati devono prender parte alla manifestazione di una emicrania diverse cause parziali. La base ereditaria sembra data da una sregolazione diencefalo-vegetativa, mentre il manifestarsi delle forme cliniche viene determinato da ulteriori influenze preforman$t i$, endogene ed esogene, di natura molto variabile.

\section{RÉSUMÉ}

On relate sur I9 cas de jumeaux souffrants de migraine et sur des constatations effectuées sur I3 couples, qu'on avait contrôlés véritables jumeaux, (trois $\mathrm{MZ}$ et dix DZ).

Pour ce qui concerne la migraine typique on a trouvé concordance seulement sur I couple $\mathrm{DZ}$ et une faible concordance sur toutes les couples $\mathrm{MX}$ et sur 3 $\mathrm{DZ}$. Mais à l'égard d'une caractéristique distonie végétative on a trouvé concordantes toutes les couples $\mathrm{MZ}$ et $2 \mathrm{DZ}$. Suivant ces constatations on peut conjecturer, que plusieures causes partielles doivent participer à la manifestation d'une migraine.

La base héréditaire paraît constituée par une régulation diencéphale-végétative, tandis que la manifestation des formes cliniques est déterminée par d'ultérieures influences préformantes, endogènes et exogènes de nature très variable.

\section{SUMMARY}

In this paper an unselected series of 19 twins with migraine is reported. The findings in 13 pairs with ascertained twin diagnosis, $3 \mathrm{MZ}$ and Io $\mathrm{DZ}$, are preliminarily summarized. With regard to typical migraine, only I $M Z$ pair was concordant, a minor degree, of concordance was seen with all $M Z$ and $3 \mathrm{DZ}$ pairs. Regarding a distinct picture of vegetative dystonia, all $\mathrm{MZ}$ and $2 \mathrm{DZ}$ pairs were concordant. On the basis of these results it can be assumed that various etiological factors are responsible for the manifestation of hemicrania. The hereditary basis seems to lie in a vegetative, diencephalic dysregulation. Besides, the clinical manifestion is determinated by various preformative influences of an endogenous or exogenous nature. 